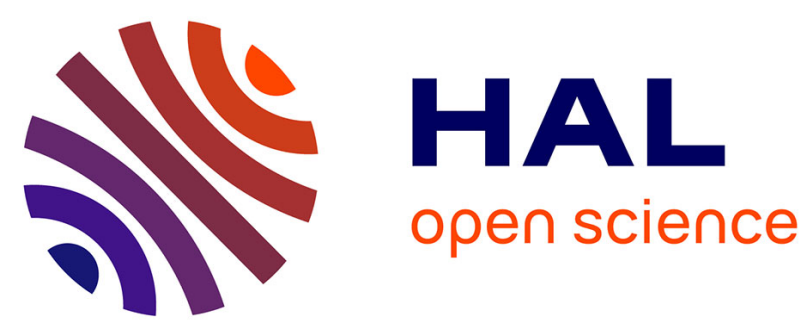

\title{
MULTI-OBJECTIVE STRUCTURAL OPTIMIZATION UNDER STRESS CRITERIA BASED ON MIXED PLATE FEM AND GENETIC ALGORITHMS
}

Pierre Garambois, Sébastien Besset, Louis Jézéquel

\section{- To cite this version:}

Pierre Garambois, Sébastien Besset, Louis Jézéquel. MULTI-OBJECTIVE STRUCTURAL OPTIMIZATION UNDER STRESS CRITERIA BASED ON MIXED PLATE FEM AND GENETIC ALGORITHMS. COMPDYN 2015, 5th ECOMAS Thematic Conference on Computational Methods in Structural Dynamics and Earthquake Engineering, May 2015, Hersonissos;Crete Island, Greece. hal01266884

\section{HAL Id: hal-01266884 \\ https://hal.science/hal-01266884}

Submitted on 3 Feb 2016

HAL is a multi-disciplinary open access archive for the deposit and dissemination of scientific research documents, whether they are published or not. The documents may come from teaching and research institutions in France or abroad, or from public or private research centers.
L'archive ouverte pluridisciplinaire HAL, est destinée au dépôt et à la diffusion de documents scientifiques de niveau recherche, publiés ou non, émanant des établissements d'enseignement et de recherche français ou étrangers, des laboratoires publics ou privés. 


\title{
MULTI-OBJECTIVE STRUCTURAL OPTIMIZATION UNDER STRESS CRITERIA BASED ON MIXED PLATE FEM AND GENETIC ALGORITHMS
}

\author{
Pierre Garambois $^{1}$, Sebastien Besset ${ }^{1}$, and Louis Jézéquel ${ }^{1}$ \\ ${ }^{1}$ Laboratoire de Tribologie et Dynamique des Systèmes, UMR CNRS 5513 \\ Ecole Centrale de Lyon, 36 avenue Guy de Collongue, 69134 Ecully Cedex, France \\ e-mail: \{pierre.garambois,sebastien.besset,louis.jezequel\}@ec-lyon.fr
}

Keywords: Structural optimization, multi-objective optimization, mixed FEM, Genetic Algorithms(GA), stress criteria

\begin{abstract}
The purpose of this study is to implement a powerful multi-objective (MO) genetic algorithm(GA)-based optimization technique for structural problems, based on the use of displacement-generalized stress Dynamic Mixed plate Finite Element Model (DMplate-FEM). The idea is to optimize different thickness parameters of a plate structure in order to minimize antagonist objectives among these stress criteria under dynamic loads. In this article, the optimization is performed with a GA-based iterative method and propose a set of Pareto-optimal solutions, leaving the final choice to the user. The repetitions of the calculations in this method is offset by the use of the displacement-stress mixed plate FEM. Such a model discretizes both displacements and generalized stresses in the same model, in order to get the latter directly and increase the efficiency of the method. The implementation of the Kirchoff-Love (KL) plate theory also improve the work of the GA as it allows a quick change of the thickness structure parameters without rebuilding the whole assembly. The number and complexity of calculations needed by the GA is thus smaller and the speed of the method highly improved. A few numerical investigations are made with both simple and complex test examples in order to validate the methods and show its relevance. This work shows efficient and promising results that may turn out to be interesting regarding industrial optimization cases with plate structures.
\end{abstract}




\section{Introduction}

The purpose of this study is to implement a powerful MO-GA-based optimization technique for structural dynamic plate problems, based on the use of mixed displacementgeneralized stress plate FEM. This method has been imagined in the context of plate structure with at least one stress objective and thickness parameters.

The optimization of a structure's parameters in order to prevent damages is a major concern in mechanical engineering but can only be achieved at the expense of others essential criteria. Even though often carried out manually, MO-optimization methods also naturally appear in the literature, such as gradient-based method [1, 2] and MOGA-based methods [3, 4]. In this study, the optimization is performed with a classical GA-based NSGA-II method [5, 6], as it permits to dispense with any weighting of criteria. This kind of method enables to find a set of Pareto-optimal solutions, which are all optimal compared to each other, for at least one criterion. Nevertheless, an inconvenient lies in the repetitions of the criteria's evaluation, depending on the chosen method and theory.

Despite the power of those methods, a major inconvenient lies in the repetitions of the criteria's evaluations for each solution, each generation. In the case where some of the criteria are stresses, their calculations are crucial. Usually, it necessitates to find the displacements first through a primal FEM (in which the parameters are displacements), and then access to the stress fields with extra calculations. Another original method, used in this paper, consists in building a mixed FEM which discretizes both the displacements and the stresses in the same model, in order to get the latter directly.

In the literature, many different formulations for mechanical problems permit to access to different parameters in the same model. Washizu's book [7] gives a good insight of all the variational methods with different fields. Among these, the Hellinger-Reissner's, imagined by Hellinger [8] and later by Reissner [9, 10, 11], describes both displacements and stresses. Its application to FEM is well explained by Wriggers' book [12]. It is widely used in static for plate problems, but more rarely for in dynamics [13, 12, 14]. In this work, we establish a displacement-generalized stress dynamic mixed FEM (DM-FEM) for structural studies, based on Kirchoff-Love thin plate theory [15]. This model allows a direct access to the generalized stresses within the plate, and highly simplifies the work of the GA under stress criteria, compared to a regular primal displacement FEM that need an extra work.

Moreover, the plate theory implementation gives us the opportunity to act on the thicknesses of the plate structure without building a whole new assembly, which offset the numericaly bigger size of such a model. This also improves the performances of the optimization techniques with some thickness parameters as we can act on them directly and independently, and change the whole structure in a simple manner.

In this way, a few investigations are made, using a GA-based optimization improved by the DM-plate-FEM, with stress criteria and thickness parameters. The idea is to split the test structure into smaller ones with their own thicknesses, and try to optimize them, so as to reduce the maximum Von Mises (VM) stress within the plate structure under a dynamic harmonic load, and the mass of the structure.

This original method shows promising results as it is able to satisfy multiple damage criteria with different thickness distributions within the structure, and decrease the number and complexity of calculations needed in the iteration of the algorithm thanks to the DM-FEM. 
In the first part, we present the type of optimization problem we study. Then we give a quick description of the GA used. We continue with the implementation of the mixed FEM, and finally get to the principal relevant examples.

\section{Optimization problem}

The two "example" structures we want to optimize are built up with thin KirchhoffLove plate elements (see figure 1 and 2, respectively composed of 256 and 512 elements). They are separated in a few zones with their own thicknesses (respectively 8 and 7 zones in the figures), which are the parameters of the optimization. The range of the parameters, as well as the number of zones and their positions are defined by the user.

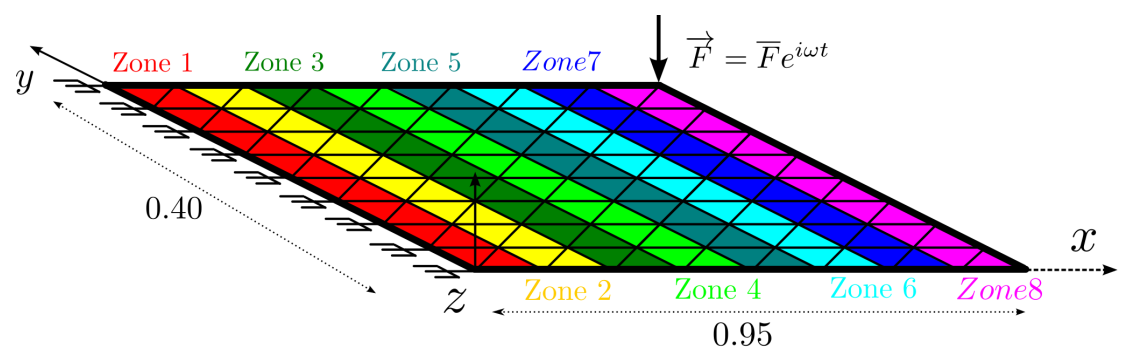

Figure 1: Plate structure "1" composed of 8 sub-structures with their own thicknesses

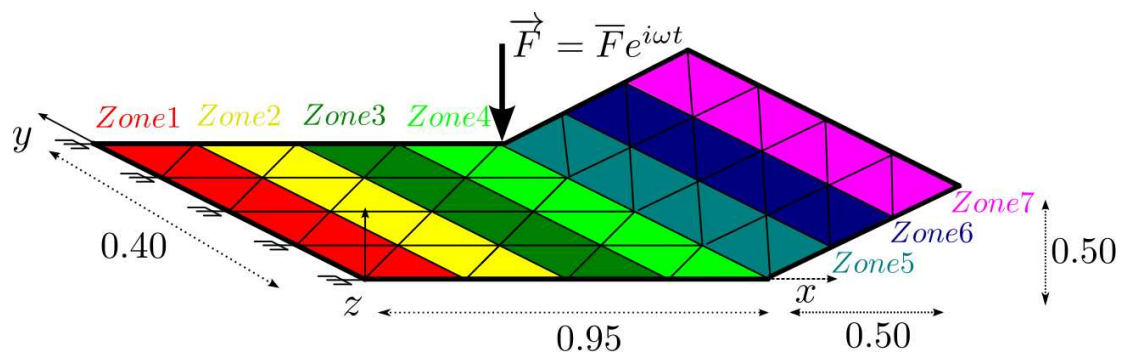

Figure 2: Plate structure "2" composed of 7 sub-structures with their own thicknesses

The problem we try to solve is the minimization of both:

- the maximum Von Mises (VM) stress within the whole plate structure for a given $n^{\text {th }}$ mode. In other words, we want to decrease the maximum VM stress within the whole structure under a dynamic load $\vec{F}$ (see figure 1 and 2 ) considering:

$$
\vec{F}=\bar{F} e^{i \omega t}
$$

and $\omega=\omega_{n}$ the pulsation of the $n^{\text {th }}$ mode

- the mass of the structure, in a bid of cost

These two objectives being antagonistic, the goal is to find some thickness distributions (set of parameters) that are good compromises between the mass and the maximum stress undergone by the structure. As such, the GA method quite naturally appears. 


\section{Genetic Algorithm method}

The GA methods can be compared to the evolution of species in their natural environment. It consists in making evolve a population, whose individuals, solutions of the problem, tend to improve for the purpose of our objectives as the generations follow. In this context, the word used to explain its functioning are taken from biology domain, namely:

- an "individual" is a solution to the problem (here, a combination of " $n$ " thicknesses (one each zone) that gives a compromises between the objectives)

- a "population" is a group of "individuals" (here, a set of thickness combinations, that forms a Pareto front with one compromises per thickness distribution)

- a "generation" is an iteration of the algorithm and correspond to a population

The functioning of the algorithm is summarized in figure 3). It is composed of 5 different stages (stages 2 being the most costly).

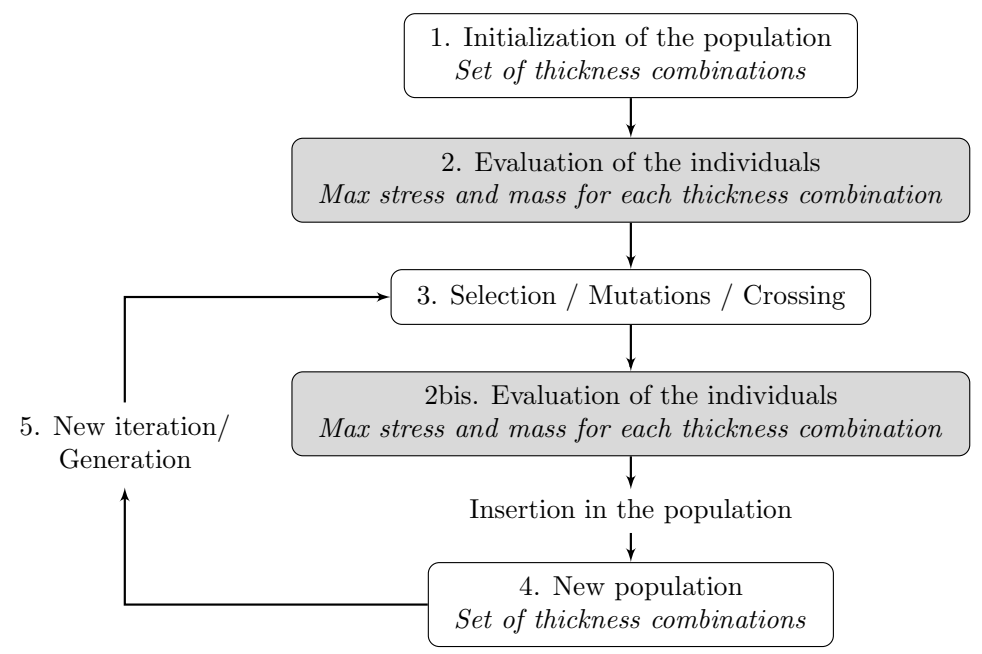

Figure 3: Principle of the genetic algorithm

\section{Dynamic Mixed Finite Element Model for thin Kirchhoff- Love plate}

In our studies, the "evaluation step" (step 2 in figure 3) is by far the most costly because we need to evaluate the stress within the whole plate structure, identify the maximum, and it eventually necessitate to rebuild the assembly with the new structure parameters. The classical method consists in building a primal FEM to access the displacement in a first step when computing the response of the structure. Then we reach the stress field through matrix calculations, and repeat it each iteration. It's the most common and intuitive method, but it appears to be heavy going because of the extra calculations, the rebuilding and its repetition in our GA case. The main idea of this paper is to delete those 
extra calculations and enhance the rebuilding. The originality of our method consists in the construction of a displacement-generalized stress DM-plate-FEM that permits a direct access to both displacements and stress fields in the response of the structure. The features of such a FEM permits to avoid the extra work needed by a primal FEM and replace it by a simple scalar calculation. Furthermore, the implementation of the Kirchhoff-Love thin plate theory in the mixed FEM allows to build each zone of a constant thickness regardless of this inherent parameter, when separating the bending phenomenon. That added features permits to act on the thickness of each zone without having to rebuild it from the beginning which considerably improve the method.

\subsection{Hellinger-Reissner mixed formulation}

The displacement-generalized stress mixed FEM we implement is based on the HR mixed functional [8, 9] expressed for dynamics problems. It may correspond to the regular Lagrangian used in dynamics, but computed with mixed component, both functions of displacements and stresses, as follows:

$$
\Pi_{H R D}=\iiint_{V}-\boldsymbol{\sigma}_{i j} \boldsymbol{e}_{i j}\left(\boldsymbol{u}_{i}\right)+\frac{1}{2} \boldsymbol{\sigma}_{i j} \boldsymbol{S}_{i j k l} \boldsymbol{\sigma}_{k l}+\boldsymbol{b}_{i} \boldsymbol{u}_{i}+\frac{1}{2} \rho \dot{\boldsymbol{u}}_{i}^{2} \mathrm{dV}
$$

considering $\boldsymbol{\sigma}_{i j}$ the stress, $\boldsymbol{u}_{i}$ the displacement, $\boldsymbol{e}_{i j}\left(\boldsymbol{u}_{i}\right)$ the strain (function of the displacement $\left.\boldsymbol{u}_{i}\right), \boldsymbol{b}_{i}$ the body force, $\rho$ the density and $\boldsymbol{S}_{i j k l}$ the elastic compliance matrix. The stationary condition or Euler-Lagrange equations can then be applied to the functional so as to conventionally solve a dynamic structure problem.

\subsection{Discretization of displacements and generalized stress}

The discretization of the generalized stresses (according to figure $4 \mathrm{a}$ ) with plate elements using Kirchhoff-Love (KL) thin plate is made as follows:

$$
\boldsymbol{\sigma}_{i j}=\left\{\begin{array}{lll}
M_{x} & M_{y} & M_{x y}
\end{array}\right\}^{T}=\boldsymbol{P} \boldsymbol{\beta}
$$

with $\left\{M_{x}, M_{y}, M_{x y}\right\}^{T}$ respectively the bending moments in the $x$, and $y$ direction, and the twisting moment within the plate (thickness $t$ ). $\boldsymbol{P}$ is the generalized stresses shape function matrix and $\boldsymbol{\beta}$ the generalized stress parameters vector within the plate. The shape functions are linear in functions of $x$ and $y$. The discretization of the displacements (see figure 4) b for a thin plate elements using Kirchhoff-Love (KL) theory is made as follows:

$$
\boldsymbol{u}_{i}=\left\{\begin{array}{lll}
w & \theta_{x} & \theta_{y}
\end{array}\right\}^{T}=\boldsymbol{N} \boldsymbol{U}
$$

with $\left\{\begin{array}{lll}w & \theta_{x} & \theta_{y}\end{array}\right\}^{T}$ being respectively the transverse displacement of the plate, and the section rotations around $x$ and $y . \quad N=\left\{\boldsymbol{N}_{w}, \boldsymbol{N}_{\theta}\right\}^{T}$ is the displacements shape function matrix and $\boldsymbol{U}=\left\{\boldsymbol{U}_{w}, \boldsymbol{U}_{\theta}\right\}^{T}$ the displacements parameters vector. For triangular elements and cinematically admissible displacements, the functions are quadratic in function of $x$ and $y$. The strain depending on the displacement is written as follows:

$$
\boldsymbol{e}_{i j}=\left\{\begin{array}{lll}
\epsilon_{x x} & \epsilon_{y y} & \gamma_{x y}
\end{array}\right\}^{T}=\boldsymbol{D} \boldsymbol{u}_{i}=\boldsymbol{D} \boldsymbol{N} \boldsymbol{U}
$$




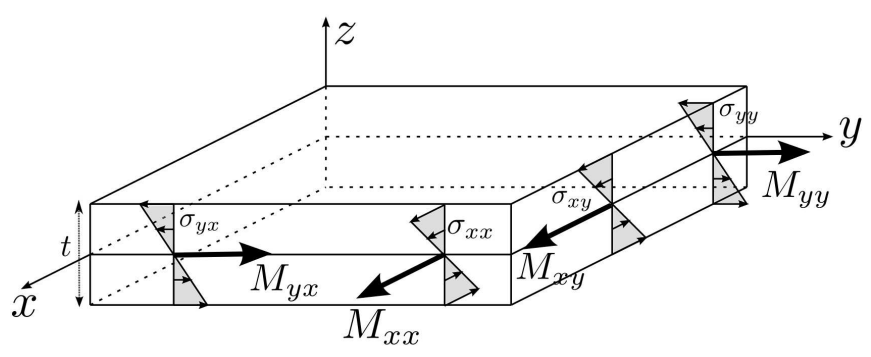

(a)

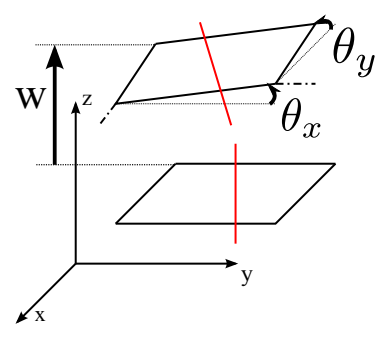

(b)

Figure 4: Displacements \& stress in the plate Kirchhoff-Love theory: (a) Bending and twisting moment, (b) Transverse displacement and rotation section

with $\boldsymbol{D}$ the following displacement-strain tensor-operator:

$$
\boldsymbol{D}=\left\{\begin{array}{ccc}
0 & 0 & \frac{\partial}{\partial x} \\
0 & -\frac{\partial}{\partial y} & 0 \\
0 & \frac{\partial}{\partial x} & \frac{\partial}{\partial y}
\end{array}\right\}
$$

The elastic compliance matrix $\boldsymbol{S}_{i j k l}$ is given by:

$$
\boldsymbol{S}_{i j k l}=\frac{1}{t^{3}} \tilde{\boldsymbol{S}}_{i j k l}=\left\{\begin{array}{ccc}
\frac{12}{E t^{3}} & -\frac{12 \nu}{E t^{3}} & 0 \\
-\frac{12 \nu}{E t^{3}} & \frac{12}{E t^{3}} & 0 \\
0 & 0 & \frac{24(1+\nu)}{E t^{3}}
\end{array}\right\}
$$

\subsection{Matrix formulation}

The application of the Euler-Lagrange equations leads us to the following matrix formulation of the dynamic mixed FEM (for one plate element of surface $S$ and thickness $t$ ):

$$
\underbrace{\left\{\begin{array}{ccc}
t \tilde{\boldsymbol{M}}_{F_{w}} & \mathbf{0} & \mathbf{0} \\
\mathbf{0} & t^{3} \tilde{\boldsymbol{M}}_{F_{\theta}} & \mathbf{0} \\
\mathbf{0} & \mathbf{0} & \mathbf{0}
\end{array}\right\}}_{\boldsymbol{M}_{m i x}}\left\{\begin{array}{c}
\ddot{\boldsymbol{U}}_{F_{w}} \\
\ddot{\boldsymbol{U}}_{F_{\theta}} \\
\ddot{\boldsymbol{\beta}}
\end{array}\right\}+\underbrace{\left\{\begin{array}{ccc}
\mathbf{0} & \mathbf{0} & \boldsymbol{G}_{w}^{T} \\
\mathbf{0} & \mathbf{0} & \boldsymbol{G}_{\theta}^{T} \\
\boldsymbol{G}_{w} & \boldsymbol{G}_{\theta} & \frac{1}{t^{3}} \tilde{\boldsymbol{H}}
\end{array}\right\}}_{\boldsymbol{K}_{m i x}}\left\{\begin{array}{c}
\boldsymbol{U}_{F_{w}} \\
\boldsymbol{U}_{F_{\theta}} \\
\boldsymbol{\beta}
\end{array}\right\}=\left\{\begin{array}{c}
\boldsymbol{F}_{w} \\
\boldsymbol{F}_{\theta} \\
\mathbf{0}
\end{array}\right\}
$$

with

$$
\begin{gathered}
\tilde{\boldsymbol{M}}_{F_{w}}=\iint_{S} \boldsymbol{N}_{\boldsymbol{w}}^{T} \rho \boldsymbol{N}_{\boldsymbol{w}} \mathrm{d} \mathrm{S} \text { et } \tilde{\boldsymbol{M}}_{F_{\boldsymbol{\theta}}}=\iint_{S} \boldsymbol{N}_{\boldsymbol{\theta}}^{T} \frac{\rho}{12} \boldsymbol{N}_{\boldsymbol{\theta}} \mathrm{dS} \\
\boldsymbol{G}_{\boldsymbol{w}}=\iint_{S} \boldsymbol{P}^{T} \boldsymbol{D} \boldsymbol{N}_{\boldsymbol{w}} \mathrm{dS} \text { et } \boldsymbol{G}_{\boldsymbol{\theta}}=\iint_{S} \boldsymbol{P}^{T} \boldsymbol{D} \boldsymbol{N}_{\boldsymbol{\theta}} \mathrm{dS} \\
\tilde{\boldsymbol{H}}=\iint_{S}-\boldsymbol{P}^{T} \tilde{\boldsymbol{S}}_{i j k l} \boldsymbol{P} \mathrm{dS}
\end{gathered}
$$

with $\boldsymbol{G}=\left\{\boldsymbol{G}_{w}, \boldsymbol{G}_{\theta}\right\}, \tilde{\boldsymbol{S}}_{i j k l}=t^{3} \boldsymbol{S}_{i j k l}$ and $\boldsymbol{F}=\left\{\boldsymbol{F}_{w}, \boldsymbol{F}_{\theta}\right\}^{T}$ being the nodal forces (splitted for $w$ and $\theta$ parameters).

When carefully developing the discretization of each component of the displacements and 
generalized stresses, it appears that we can split the whole matrices into smaller ones independent on the thicknesses: $\tilde{\boldsymbol{M}}_{F_{w}}$ representing the mass in the sense of the transverse displacement $w, \tilde{\boldsymbol{M}}_{F_{\theta}}$ representing the mass in the sense of the rotation section around $x$ and $y$ (respectively $\theta_{x}$ and $\theta_{y}$ ) and $\tilde{\boldsymbol{H}}$ representing the stiffness in the meaning of the stress parameters. $\boldsymbol{G}$ is intrinsically independent from the thickness of the element. That features permits to build an assembly of a given structure (or specific zone of it in our case) regardless of its thickness (as long as it is constant in the zone). Thus, we can build the assembly of each $q$ zone of the structure, attribute their own thicknesses, and afterwards assemble the $q$ zones in order to build the "total" assembly.

In the rest of the article, the superscripts..$^{(q)}$ and.$^{\text {tot }}$ are added to the matrices in order to make the distinction between respectively the matrices of the zone $q$ and the ones of the "total" structure.

\subsection{Computation of the response and the Von Mises stress}

The computation of the response of the "total" structure is made with a global viscous damping proportional to the stiffness features, as follows:

$$
\boldsymbol{C}_{m i x}^{t o t}=\alpha \boldsymbol{K}_{m i x}^{t o t}
$$

Thus, the mixed response of the "total" structure is computed as follows:

$$
\left\{\begin{array}{l}
\overline{\boldsymbol{U}} \\
\overline{\boldsymbol{\beta}}
\end{array}\right\}=\left(-\omega^{2} \boldsymbol{M}_{\text {mix }}^{\text {tot }}+(1+i \alpha \omega) \boldsymbol{K}_{\text {mix }}^{t o t}\right)^{-1}\left\{\begin{array}{c}
\overline{\boldsymbol{F}} \\
0
\end{array}\right\}
$$

with $\omega=\omega_{n}$ the pulsation of the $n^{\text {th }}$ mode in our case. The VM stress in then computed as follows:

$$
\sigma_{V M}=\frac{1}{\sqrt{2}} \sqrt{\left(\sigma_{x x}-\sigma_{y y}\right)^{2}+\sigma_{y y}^{2}+\sigma_{x x}^{2}+6 \sigma_{x y}^{2}}
$$

\subsection{Implementation in the genetic algorithm, features and ad- vantages}

The figure 5 shows the implementation of the DM-FEM in the GA, with a population of $p$ individuals, $g$ generations, and $q$ different parameters (zones' thickness). This type of mixed model coupled with the thin plate theory puts forward two clear advantages comparing to primal FEM:

- a direct access to the generalized stress within the plate (see operation "2.c" in schema figure 5) and the suppression of the extra calculation needed by a primal FEM. Basically, we replace a complex matrix calculation (for example with Gauss points and smoothing methods) by a simple scalar and almost instantaneous calculation (equation 14). Even though the response calculation (equation 13) is a bit heavier than for a primal model because of its size, the global operation remains more efficient with a mixed model

- the thickness parameters of each $q$ zone of the structure are easily mutable (operation "2.a" in schema figure 5), before assembling them (operation 2.b in schema figure 
5). Even though such models are numerically bigger than a classical FEM, the "preassembly" of $\boldsymbol{M}_{m i x}^{(q)}$ and $\boldsymbol{K}_{m i x}^{(q)}$ for each $q$ zone regardless of the thickness parameters (operation 0 in schema figure 5) leaves us the opportunity to allocate them separately for each $q$ zone each iteration (operation "2.a") and afterwards assemble the "total" structure's matrices $\boldsymbol{M}_{m i x}^{\text {tot }}$ and $\boldsymbol{K}_{m i x}^{\text {tot }}$ (operation 2.b ). The replacement of a "global assembly" by a simple assembly of $q$ zones, each iteration each generation, highly increases the performance of the method.

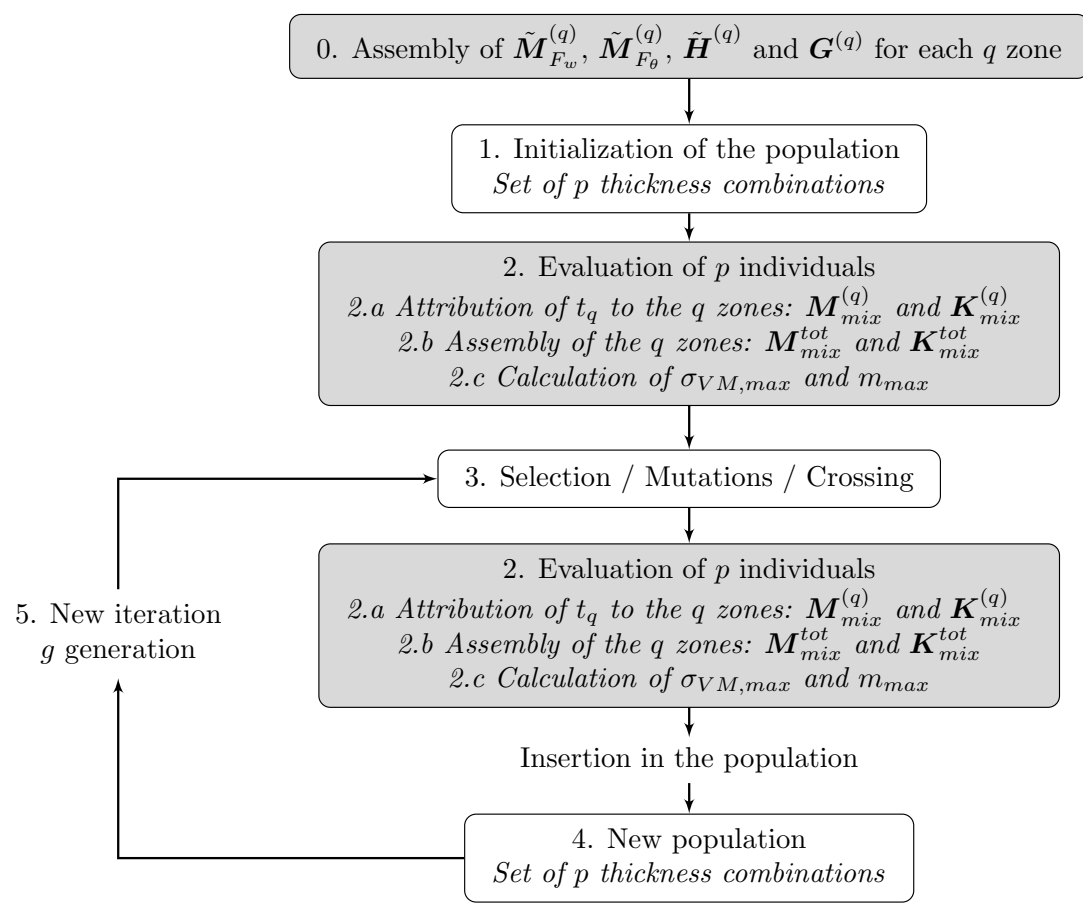

Figure 5: Implementation of the mixed FEM in the genetic algorithm, with a population of $p$ individuals, $g$ generations, and $q$ different parameters (zones)

\section{Example: optimization of the thickness combina- tion to minimize the mass \& the maximum stress for one selected mode}

The examples we use to test our method are schematized in figure 1 and 2 in section 2. We want to minimize the maximum VM stress $\omega_{V M}$ within the whole structure for a given mode $n$ (see figure 6. c, 7.c and 8. $/ \mathrm{f}$ ), and the mass $m$ of it. The parameters we consider are the thicknesses of each "zone" (see thickness zones in figures $6 \mathrm{~b}, 7 \mathrm{~b}$ and $8 \mathrm{~b} / \mathrm{e}$ ). The range chosen for the different parameters is 1 to $2 \mathrm{~mm}$. The plate is made of $S 210$ steel (see characteristics in table 1).

The results are presented as "Pareto Front" (black points in figures 6. a, 7,a and 8a/d). These points are defined by the right ordinate axis and the abscissa axis representing respectively the mass of the structure and the maximum VM stress. Each of these optimal 
Table 1: Steel characteristics

\begin{tabular}{cc}
\hline Young Modulus $(\mathrm{Pa})$ & $2.1 \times 10^{11}$ \\
\hline Poisson ratio & 0.33 \\
\hline Density $\left(\mathrm{kg} \cdot \mathrm{m}^{-3}\right)$ & $7.5 \times 10^{3}$ \\
\hline
\end{tabular}

points are better than the others in the sense of at least one of the two objectives. In that way they are all compromises we can select to design our structure, depending on the objective we want privilege. Once a Pareto point chosen, the parameters corresponding to the point are defined by the colored curves plotted in addition to the Pareto Front in figures 6, a, 7, a and 8 $\mathrm{a} / \mathrm{d}$. They are determined by the left ordinate axis and the abscissa axis representing respectively the thickness of each of the sub-structures for the chosen Pareto point (with the same abscissa) and the maximum VM stress in the whole structure for this optimal point. Basically, when selecting a point of the Pareto Front, the corresponding thicknesses to design the structure correspond to ordinate of the colored three points with the same abscissa.

Those results were obtained with NSGA-II with a population of $p$ individuals and $g$ generations (depending on the case) following the schema figure 5. It is to be noted that the genetic algorithm has been compiled several times with the same population and generation, in order to check the convergence of the results.

The first subsection deals with the optimization of structure 1 for the mode 1 and constitutes a simple example whose sketch of the results could easily be intuitive, while the second one treats of the optimization of the more complex structure 2 for the mode 3 . in the end, subsection 3 shows results for the mode 4 of structure 1 and tricky results.

\subsection{Structure 1, Mode 1: Validation / Simple example}

This subsection shows a simple intuitive example with structure 1, in order to show the qualitative relevance of such a method. It presents the results obtained with the pulsation $\omega_{1}$ of the mode $n=1$ (bending mode), a damping coefficient $\alpha=2 e^{-2}$ and for a stress distribution divided in $q=8$ zones. The structure is composed of 256 elements. In the genetic algorithm, the population is composed of $p=50$ individuals and the results presented in figure 6 are obtained with $g=500$ generations.

This example shows some thickness combinations that allows us to have a VM stress between 17 and $142 \mathrm{MPa}$, with a mass of the structure between 2.85 and $4.8 \mathrm{Kgs}$. In this case, more than the steadiness of the Pareto Front itself, the most relevant is the evolution of the thicknesses in function of the VM stress (see colored curves in figure 6a). In fact, we can observe that the most important zones to "reinforce" in order to decrease damages are the closest to the housing. Indeed, the closer to the housing the zone is, the more it needs to be thick. When taking a look at the stress distribution for the first mode (see figure 66), this evolution of the thicknesses in function of the stress seems coherent. Intuitively, in order to decrease the mass and keep a low stress, we would have decrease 


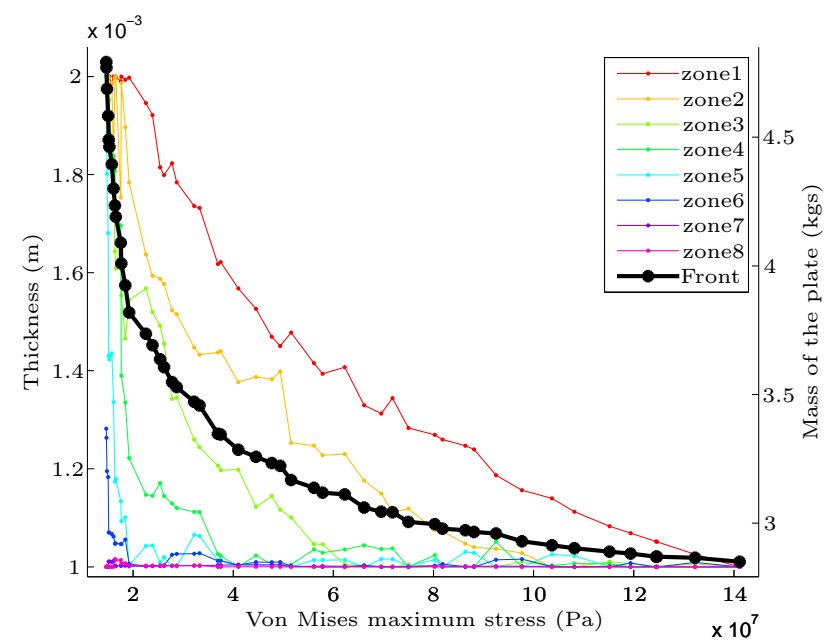

(a)

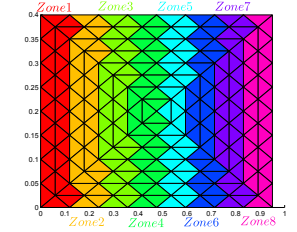

(b)

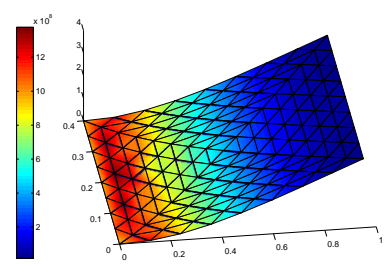

(c)

Figure 6: Optimization results for "structure 1" and mode 1: a/ Pareto Front (black) \& thicknesses $(\mathrm{m})$ for each point (colored), b/ Independent thickness zones, c/ Stress distribution on mode $1(\mathrm{~Pa})$

the thickness where the stress is low, and increase it where it is high. This example puts forward the qualitative relevance of the method, and also gives a quantitative aspect to the thickness parameters with numerical precise values.

\subsection{Structure 2, Mode 3: Validation / Complex example}

This subsection presents intuitive results with a less simple example with the structure 2 (see figure 2). It presents the results obtained with the pulsation $\omega_{3}$ of the mode $n=3$ (bending mode), a damping coefficient $\alpha=2 e^{-3}$ and for a stress distribution divided in $q=10$ zones. The structure is composed of 512 elements. In the genetic algorithm, the population is composed of $p=100$ individuals and the results presented in figure 7 are obtained with $g=500$ generations.

This example shows some thickness combinations that allows us to have a VM stress between 14 and $142 \mathrm{MPa}$, with a mass of the structure between 4.95 and $8.3 \mathrm{Kgs}$. In the same way as section 5.1, the most interesting is the evolution of the thicknesses in function of VM stress (see colored curves in figure $7 \mathrm{a}$ ). In fact, when observing the stress distributions for this mode (see figure $7 \mathrm{k}$ ), we note that the most stressed zones (see figure 7b) are the zones 1, 5, 6 and 7. It seems intuitive to reinforce these zones specifically in order to decrease the VM stress within the structure. Indeed, the evolutions of the thicknesses $7 \mathrm{a}$ ) follows this intuition, as these zones look to be the most important, stress wise. In this way, this example, even though more complex than the first one, also puts forward the relevance of the method, in a qualitative manner.

Besides the evolution of the stress distribution that is intuitively predictable, as for the last example, our method still add a qualitative interest as figure $7 \mathrm{a}$ gives precise values to the thickness parameters we need to select in order to get the corresponding maximum VM stress and mass of the selected point. 


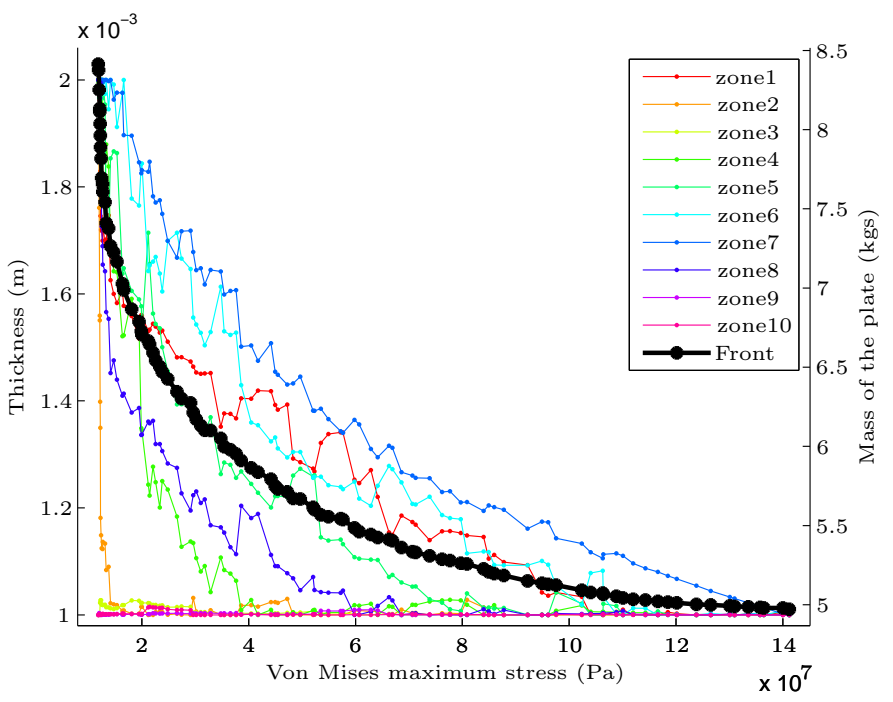

(a)

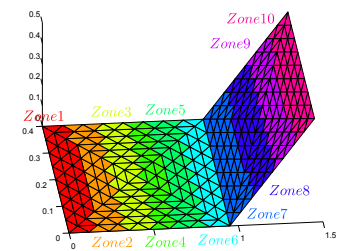

(b)

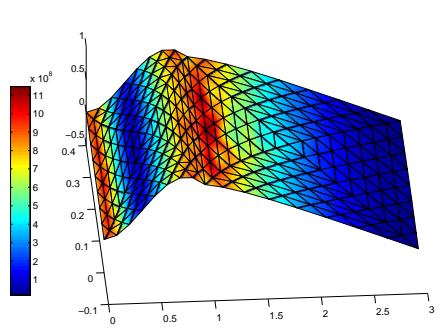

(c)

Figure 7: Optimization results for "structure 2" and mode 3: a/ Pareto Front (black) $\&$ thicknesses $(\mathrm{m})$ for each point (colored), b/ Independent thickness zones, c/ Stress distribution on mode $1(\mathrm{~Pa})$

\subsection{Structure 1, Mode 4}

This section focus on the $2^{\text {nd }}$ twisting mode of the structure 1 . It presents the results obtained with the pulsation $\omega_{4}$ of the mode $n=4$ (twisting mode), a damping coefficient $\alpha=1 e^{-4}$. We implemented two cases both divided in 4 zones, with a different repartition of the zones (see figure $8 \mathrm{~b}$ and $8 \mathrm{~b}$ ). The results are summed up for this two configurations respectively in figure $8 \mathrm{a}$ and $8 \mathrm{~d}$. In both cases, the structure is composed of 256 elements, the population is made with $p=50$ individuals and the number of generations is $g=500$.

The Pareto Front in both cases are not as well-defined and steady as for the last cases. In the first configuration, it allows us to have a VM stress between 3.4 and $170 \mathrm{MPa}$, with a mass of the structure between 2.85 and $4.23 \mathrm{Kgs}$. In the second one, the VM stress goes from 3.3 up to $170 \mathrm{MPa}$, with a mass of the structure between 2.85 and $4.24 \mathrm{Kgs}$. We note that the Pareto Front both presents some specificity we didn't observe in the optimization of the $1^{\text {st }}$ mode (see section 6), as in both cases:

- the Pareto front shows a large set of solutions that have almost the same VM stress for wide range of mass (low stress domain). This domain is interesting stress wise, because it allows the user to choose this low stress, and dispose of a many different solutions parameters wise. We could call that phenomenon "stress typing"

- the Pareto front shows a large that have a mass between 3.35 and $2.85 \mathrm{kgs}$ for the first configuration and 3.46 and $2.85 \mathrm{kgs}$. In this low mass domain, the VM stress varies a lot between respectively 6.6 and $170 \mathrm{MPa}$, and 6.8 and $170 \mathrm{MPa}$. This domain is interesting mass wise, because it allows the user to choose a low mass in this domain, and dispose of a a large set of parameters possibility. We could call that phenomenon "mass typing" 


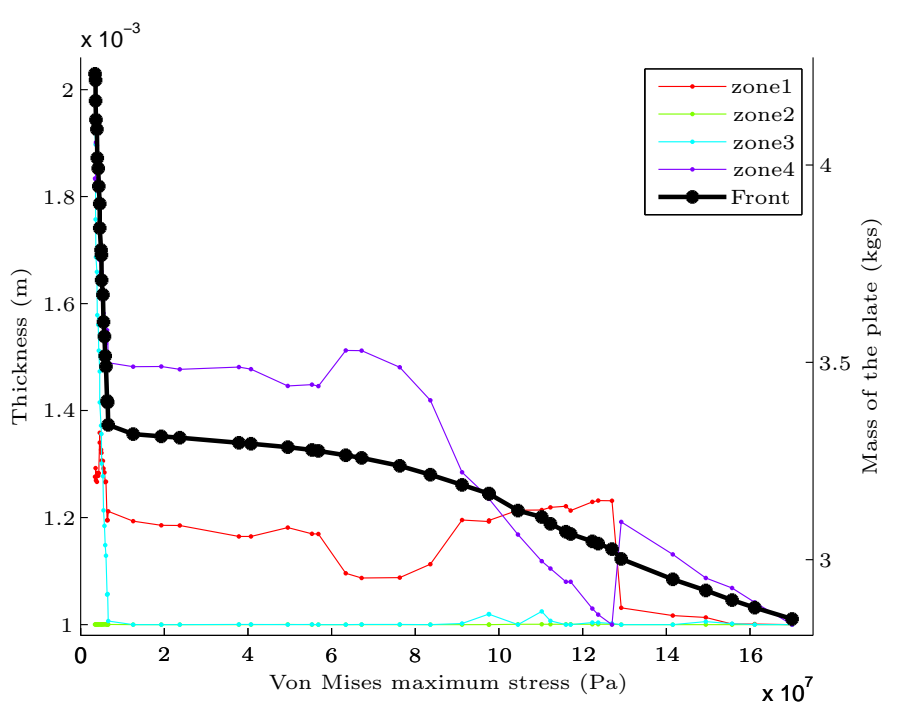

(a)

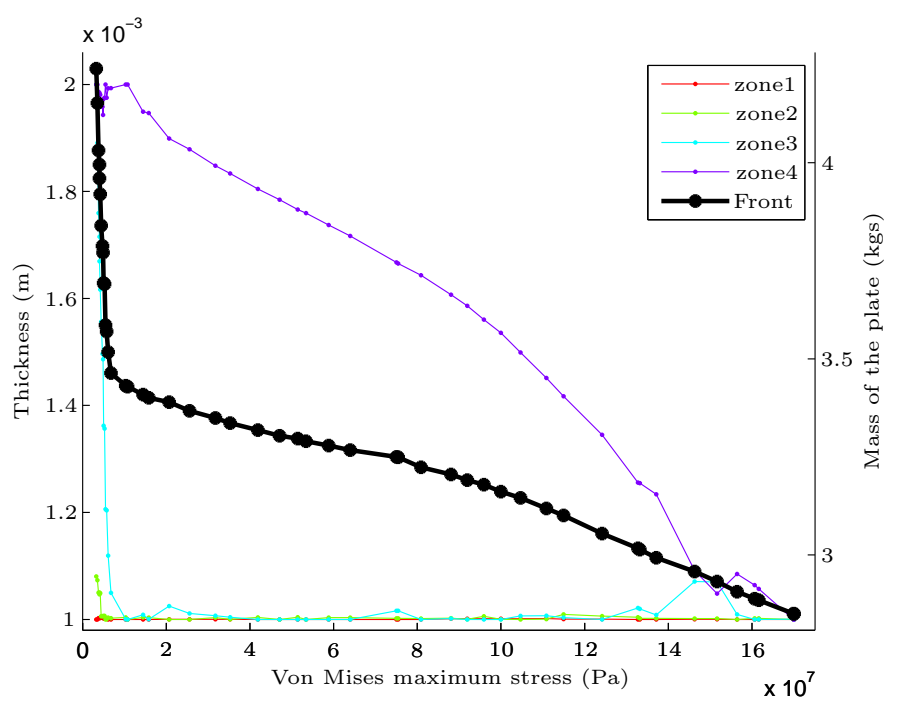

(d)

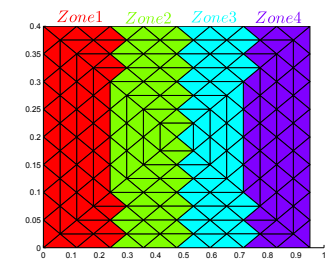

(b)

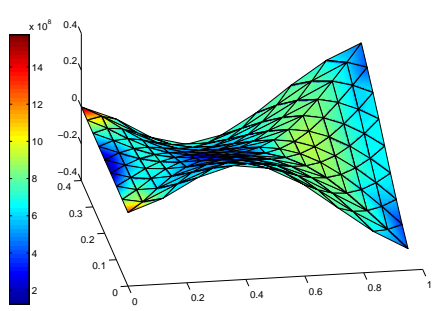

(c)

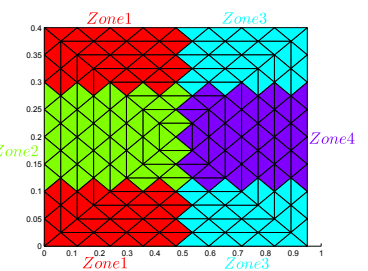

(e)

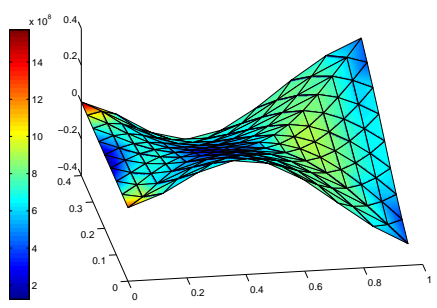

(f)

Figure 8: Optimization results for "structure 1" and mode 5: a/ Pareto Front (black) \& thicknesses (mm) for each point (colored), b/ Independent thickness zones, c/ Stress distribution on mode $5(\mathrm{MPa})$ 
When observing the stress distribution within the plate for the $4^{\text {th }}$ mode (see figure 8k/f), it seems tricky to define intuitively which zones need reinforcement. Nevertheless, a first guess would be to increase the thickness either of the first zone (red) in the second configuration (figure 8 $8 \mathrm{~d} / \mathrm{e}$ ) or the first zone (red) in the first configuration figure $8 \mathrm{a} / \mathrm{b}$ ). Nevertheless, the two Pareto front show different results, as the second configuration prescribes to pay attention to the $4^{\text {th }}$ central violet zone (almost exclusively), whereas the first configuration focus more on its $4^{\text {th }}$ violet zone and a bit on the $1^{\text {st }}$ red one. Furthermore, the first configuration shows a parameters typing with a switching between the $1^{\text {st }}$ and $4^{\text {th }}$ zones in the high stress domain. This phenomenon can present a high interest in an industrial case as it means that different set of parameters leads to the same stress and mass configuration.

The results described here puts forward the importance of the choice of the distribution of the zone at the beginning of the study, and the relevance of the method in non-intuitive cases. These unexpected results could be interesting in a complex non-predictable industrial case, and puts forward the interest of such a method, in addition to the simplicity and efficiency highlighted in section .

\section{Conclusion}

This paper introduces a methodology of MO optimization for structural dynamic plate problems with stress criteria and thickness parameters. It is based on a classical NSGA-II method based on compromises and Pareto-optimal solutions, but presents the particularity of using a displacement-generalized stress DM-plate-FEM. Such a model permits a direct access to the stress criteria compared to a primal FEM that needs extra complex calculation. Furthermore, when implementing a KL plate theory, this mixed FEM is featured by a linear dependence to the thickness parameters which facilitates the work of the repetitive algorithm as a whole new assembly is not necessary. These two features combined permits to improve the costly "evaluation step" of the GA and to make it more efficient than classical methods.

This article shows three practical cases, using two different plate structures under a dynamic harmonic load. They are divided into a few zones and each of them has a thickness that is a parameter of the optimization. The goals of the studies are to minimize the maximum VM stress and the mass of the whole structure in function of the thicknesses of each zone, for a harmonic dynamic load of a selected mode. Our optimization technique permits to find a whole group of compromises between the maximum stress and the mass for different thickness distributions, with a different a simpler FEM model. The first examples focus on two different structures and show simple results that could be found intuitively. They put forward the qualitative relevance of the model for a simple example and a more complicated one, and also add a qualitative interest of the method. The third example, deals with a twisting mode and show interesting results. In fact, the qualitative and quantitative evolutions of the parameters of the optimal points were unpredictable, and the Pareto Front presents both stress and mass typing. These kind of results could be interesting to observe in a complex industrial case, and puts forward the interest of such a simple method, in addition to the simplicity and efficiency previously highlighted. 


\section{REFERENCES}

[1] A.L Araujo, P Martins, C.M Mota Soares, Mota Soares C.A, and J Herskovits. Damping optimization of viscoelastic laminated sandwich composite structures. Structural and Multidisciplinary Optimization, 39:569-579, 2009.

[2] N.D Lagaros and V Papadopoulos. Optimum design of shell structures with random geometric, material and thickness imperfections. International Journal of Solids and Structures, 43:6948-6964, 2006.

[3] R Kudikala, K Deb, and B Bhattacharya. Multi-objective optimization of piezoelectric actuator placement for shape control of plates using genetic algorithms. Journal of Mechanical Design, 131:091007-1-11, 2009.

[4] B Paluch, M Grédiac, and A Faye. Combining a finite element programme and a genetic algorithm to optimize composite structures with variable thickness. Composite and Structures, 83:284-294, 2008.

[5] N Srinivas and K Deb. Multiobjective optimization using nondominated sorting in genetic algorithms. Journal of Evolutionary Computation, 2:221-248, 1994.

[6] K Deb, S Agrawal, A Pratap, and T Meyarivan. A fast elitist non-dominated sorting genetic algorithm for multi-objective optimization: Nsga-ii. Notes in Computer Science, 2000.

[7] Washizu. Variational methods in elasticity and plasticity, 2nd edition. Pergamon Press, 1975.

[8] E Hellinger. Die allgemeinen ansatze der mechanik der kontinua. Encyklopadie der mathematischen Wissenschaften, 4, 1914.

[9] E Reissner. On a variational theorem in elasticity. Journal of Mathematics and Physics, 29:90-95, 1950.

[10] Reissner. On a certain mixed variational theorem and a proposed application. International Journal For Numerical Methods In Engineering, 20(7):1366-1368, 1984.

[11] E Reissner. On a mixed variational theorem and on shear feformable plate theory. International Journal For Numerical Methods In Engineering, 23(2):193-198, 1986.

[12] P Wriggers and C Cartsensen. Mixed Finite Element Technologies. Applied Mathematics/Computational Methods of Engineering, 2009.

[13] M.H Omurtag and F Kadioglu. Free vibration analysis of orthotropic plates resting on pasternak foundation by mixed finite element formulation. Computers and Structures, 67:253-265, 1998.

[14] P Garambois, S Besset, and L Jézéquel. Modal synthesis applied to a reissner mixed plate finite element dynamic model. Proceedings of the 9th International Conference on Structural Dynamics, EURODYN 2014, 2014.

[15] J. N. Reddy. Theory and Analysis of Elastic Plates and Shells. 2007. 